\title{
EVALUACIÓN DE LOS FACTORES DE REDUCCIÓN DE RESISTENCIA POR DUCTILIDAD PARA ESTRUCTURAS DE MAMPOSTERÍA CIMENTADAS EN TERRENO FIRME
}

\author{
Jorge Ruiz-García ${ }^{(1)}$ y Eduardo Miranda ${ }^{(2)}$
}

\begin{abstract}
RESUMEN
Se presentan los resultados de un estudio estadístico sobre el efecto de la degradación estructural (i.e., degradación de rigidez, deterioro de resistencia y estrechamiento de los lazos de histéresis cerca del origen) en las demandas de resistencia lateral de estructuras cimentadas sobre terreno firme o roca $\mathrm{y}$, en particular, en los factores de reducción de resistencia por ductilidad, $R_{\mu}$, que permiten reducir las ordenadas de los espectros elásticos lineales para tener en cuenta el comportamiento inelástico de la estructura. Para tal fin, se considero la respuesta inelástica de sistemas de un grado de libertad con un comportamiento bilineal, para representar a las estructuras que no exhiben degradación estructural, y con un modelo histerético que permite reproducir la degradación estructural, como la observada en estructuras de mampostería al verse sometidas a cargas laterales cíclicas reversibles. Los sistemas de un grado de libertad se sometieron a un conjunto de 264 acelerogramas registrados en estaciones ubicadas en roca o terreno firme del área de California (Estados Unidos) durante 12 sismos históricos. Como parte de este estudio, se presenta la comparación de los factores de reducción de resistencia por ductilidad considerando la degradación estructural, $R_{\mu C D}$, con los factores de reducción de resistencia calculados con el modelo bilineal, $R_{\mu S D}$, y con el factor de comportamiento sísmico, $Q$ ', propuesto recientemente para su uso en la normatividad mexicana. En particular, se presto atención especial a la evaluación de la dispersión de $R_{\mu C D}$. A partir de los resultados obtenidos se concluye que, para una misma demanda de ductilidad, los factores $R_{\mu C D}$ son menores a los que se tienen en estructuras que no presentan degradación estructural en gran parte del intervalo de periodos estudiado. Por ello, las estructuras susceptibles a sufrir degradación estructural deberían diseñarse para fuerzas laterales mayores que las estructuras que no experimentan degradación estructural.
\end{abstract}

\section{SUMMARY}

A key parameter in traditional force-based earthquake resistant design of new structures is the use of strength-reduction factors that allow the reduction of linear elastic design spectra to account for nonlinear behavior during seismic ground shaking. However, most of the previous studies on strength-reduction factors have used non-degrading systems and few studies have focused their attention on the influence of structural degradation on strength-reductions factors. Thus, this study evaluates and discusses the effect of stiffness degradation, strength deterioration, and pinching on

Artículo recibido el 15 de noviembre de 2000 y aprobado para su publicación el 10 de febrero de 2003. Se aceptarán comentarios y/o discusiones hasta cinco meses después de su publicación.

(1) John A. Blume Earthquake Engrg. Ctr., Stanford Univ., Stanford, CA 94305-4020. jruizgar@stanford.edu

(2) Department of Civil and Environmental Engineering, Stanford Univ., Stanford, CA 94305-4020. emiranda@stanford.edu 
strength reduction factors for structures built on firm soil sites. For this purpose, constant-ductility strength-reduction factors were computed from the response of single-degree-of-freedom inelastic systems, subjected to a suite of 264 earthquake ground motions recorded on rock or stiff soil sites. In this investigation, the hysteretic behavior of three types of masonry structures was analytically reproduced by the well-known three-parameter model to characterize systems with significant structural degradation while the bilinear model was used to simulate structures without structural degradation. The variability of strength-reduction factors with respect to the central tendency is specially addressed. From the results of this study, it is concluded that in the short period region (i.e., periods of vibration shorter than $0.5 \mathrm{~s}$ ) mean constant ductility strength-reduction factors for degrading systems are smaller than those for non-degrading systems. Then, structures in this period region (i.e., masonry structures) with significant structural degradation should be designed for lateral forces larger than those for structures that do not exhibit structural degradation. Finally, a comparison of the strength-reduction factors for degrading systems with the force modification factors, $Q$, recently proposed for the new seismic design criteria of the Mexico City Building Code showed that $Q^{\prime}$ factors are adequate from a force-based design viewpoint.

\section{INTRODUCCIÓN}

Actualmente los reglamentos de diseño por sismo permiten la existencia de deformaciones inelásticas en edificios y otros tipos de estructuras durante sismos de gran intensidad. Como resultado de esta filosofía de diseño las fuerzas laterales que se utilizan en el diseño de las estructuras no corresponden a las necesarias para mantener a la estructura elástica durante sismos severos, sino corresponden a fuerzas reducidas. Estas fuerzas reducidas suelen ser menores, y en algunos casos mucho menores, que las necesarias para mantener a la estructura elástica.

En la mayoría de los reglamentos, incluyendo las normas mexicanas, la reducción de fuerzas se hace a través de factores de reducción de resistencia. Estos factores deben tener en cuenta la disipación de energía y el incremento del amortiguamiento por comportamiento inelástico, así como la sobrerresistencia estructural, entre otros factores. Actualmente, se reconoce que el factor de reducción de resistencia, $R$, es igual al producto de cuatro factores (Bertero et al., 1991, Uang, 1991; Miranda, 1997; Whittaker et al., 1999)

$R=R_{s} R_{\mu} R_{V G} R_{R}$

donde $R_{s}$ es un factor de reducción por sobrerresistencia; $R_{\mu}$ es un factor de reducción debido a la disipación de energía por comportamiento no lineal de la estructura; $R_{V G}$ es un factor de reducción para tener en cuenta sistemas de múltiples grados de libertad, y $R_{R}$ es un factor de redundancia. El factor $R_{S}$ se define como el cociente de la capacidad última de la estructura y su capacidad de diseño, o nominal. Estudios recientes han observado que el factor $R_{S}$ depende, principalmente, del periodo natural de vibración de la estructura y del sistema resistente ante cargas laterales. Una discusión detallada de los factores que pueden contribuir a la sobrerresistencia estructural puede encontrarse en Miranda (1997). El factor $R_{R}$ es una medida de la participación y eficiencia de los elementos estructurales para trasmitir las cargas que son sometidas a la estructura (Bertero y Bertero, 1999). 
De ahí que para obtener una buena estimación de la resistencia lateral necesaria para limitar las demandas de ductilidad a partir de espectros elásticos lineales se requiera una buena estimación de los factores de reducción de resistencia. El factor de reducción de resistencia por ductilidad (i.e., reducción en la demanda de resistencia debido al comportamiento histerético no lineal) $R_{\mu}$ se define como el cociente de la demanda de resistencia elástica a la demanda de resistencia inelástica

$$
R_{\mu}=\frac{F_{y}(\mu=1)}{F_{y}\left(\mu=\mu_{\mathrm{i}}\right)}
$$

donde $F_{y}(\mu=1)$ es la resistencia lateral de fluencia necesaria para mantener al sistema elástico durante el movimiento de terreno y $F_{y}\left(\mu=\mu_{i}\right)$ es la resistencia lateral de fluencia necesaria para mantener la demanda de ductilidad de desplazamiento, $\mu$, menor o igual a una ductilidad de desplazamiento predeterminada $\mu_{i}$ (máxima tolerable). La ec. 2 puede re-escribirse como

$$
R_{\mu}=\frac{C_{y}(\mu=1)}{C_{y}\left(\mu=\mu_{\mathrm{i}}\right)}
$$

donde $C_{y}(\mu=1)$ es el coeficiente sísmico de diseño (la resistencia lateral de diseño entre el peso total del sistema) mínimo requerido para evitar la fluencia y $C_{y}\left(\mu=\mu_{i}\right)$ es el mínimo coeficiente sísmico requerido para controlar la demanda de ductilidad de desplazamiento menor o igual a un factor de ductilidad máximo tolerable $\mu_{i}$. Para fines de diseño sismorresistente, $R_{\mu}$ corresponde a la máxima reducción en resistencia lateral que puede ser utilizada a fin de limitar la demanda de ductilidad de desplazamiento por debajo del límite máximo tolerable en una estructura que tendrá una resistencia lateral igual a la resistencia lateral de diseño. Investigaciones recientes han evidenciado que si una estructura de varios grados de libertad es diseñada con la misma resistencia lateral que un sistema de un grado de libertad con periodo de vibración igual al periodo de vibración de la estructura de varios grados de libertad, ésta puede experimentar demandas de ductilidad mayores a las del sistema de un grado de libertad. Lo anterior explica que la resistencia lateral obtenida a partir de sistemas de un grado de libertad debe ser modificada para lograr un control adecuado de las demandas de entrepiso (Miranda et al., 1999).

Las demandas de resistencia lateral, expresadas en términos de factores de reducción de resistencia por ductilidad, ha sido un tópico importante en el diseño sismorresistente. Recientemente, Miranda y Bertero (1994) presentaron una recopilación completa, en un formato común que permite su comparación directa, de estudios previos en sistemas de un grado de libertad encaminados a estimar los factores de reducción de resistencia por ductilidad. Sin embargo, la mayor parte de los estudios previos han considerado sistemas con comportamiento elastoplástico perfecto, el cual no es representativo del comportamiento de estructuras que sufren degradación de rigidez y resistencia al verse sometidas a cargas laterales cíclicas reversibles (i.e., del tipo sísmico), como es el caso de las estructuras de mampostería. Como parte de un amplio programa experimental y analítico sobre el comportamiento sísmico de muros de mampostería, Bazán y Meli (1980) estudiaron la respuesta inelástica de sistemas con un comportamiento 
representativo de cuatro estructuraciones de muros de mampostería sometidos a tres acelerogramas registrados en terreno firme, cinco periodos de vibración y ductilidad de desplazamiento igual a cuatro. Los autores observaron que las demandas de resistencia lateral en los sistemas con degradación estructural (i.e., como la que exhiben las estructuras de mampostería) son mayores que las obtenidas para un sistema elastoplástico. Asimismo, los autores concluyeron que los factores de reducción de resistencia, o de comportamiento sísmico, establecidos por la normatividad mexicana de la época eran conservadores. Posteriormente, Moroni y colaboradores (1996), realizaron un estudio analítico para evaluar el factor de reducción de resistencia por ductilidad llevando a cabo análisis dinámicos no-lineales de cuatro tipos de estructuraciones típicas de mampostería confinada, de tres a cuatro niveles, en ambas direcciones y sometidos a acelerogramas registrados durante los sismos de Viña del Mar en Chile y México en 1985. Los autores observaron que el factor de reducción disminuyó casi linealmente conforme aumentó la densidad de muros en la dirección de estudio, concluyendo que este factor depende del periodo de vibración y, en particular, de la densidad de muros en cada dirección de análisis.

Como el lector puede notar, ninguna tendencia clara del factor de reducción de resistencia por ductilidad aplicable a estructuras de mampostería fue identificada en los estudios mencionados. Por ello, el objetivo de este estudio consiste en: (1) evaluar y cuantificar la influencia de la degradación estructural (caracterizada por la degradación de rigidez, el deterioro de resistencia y el estrechamiento de los lazos histeréticos) en la estimación de las demandas de resistencia lateral de sistemas con comportamiento representativo de estructuras de mampostería; (2) obtener los factores de reducción de resistencia por ductilidad asociados a estructuras de mampostería, $R_{\mu C D}$; (3) Evaluar la dispersión del factor $R_{\mu C D}$; y (4) comparar los resultados de este estudio estadístico con el factor de comportamiento sísmico, $Q$ ', recientemente propuesto en la normatividad mexicana. Sin embargo, debe notarse que este estudio solo se limita a evaluar el efecto de la degradación estructural en $R_{\mu}$, y no se considera la evaluación de los factores $R_{s}, R_{R} \mathrm{y}$ $R_{V G}$ para estructuras que exhiben degradación estructural.

\section{COMPORTAMIENTO HISTERÉTICO DE ESTRUCTURAS DE MAMPOSTERÍA}

\section{Comportamiento experimental}

El desempeño experimental de muros aislados y sistemas acoplados, a escala natural, de mampostería sometidos a cargas laterales cíclicas reversibles ha mostrado que la rigidez inicial sufre una disminución progresiva cuya tasa de deterioro no-lineal depende, principalmente, del nivel de deformación lateral (Alcocer y Meli, 1995; Aguilar et al., 1996; Tomazevic y Klemenc, 1997). En especimenes de mampostería confinada, el deterioro inicial de la rigidez lateral se debe al fisuramiento horizontal, por flexión, de los castillos exteriores así como al reacomodo de los tabiques. La tasa de deterioro aumenta al agrietarse diagonalmente la mampostería y sufrió una disminución brusca cuando las grietas diagonales penetran a los extremos de los castillos. Una tasa de deterioro similar ha sido observada en especimenes con refuerzo horizontal interior y con refuerzo exterior (Aguilar et al., 1996; Alcocer et al., 1996). 
A partir de la respuesta histerética global observada en especimenes de mampostería confinada se puede identificar una envolvente caracterizada por tres etapas de comportamiento. La primera etapa se puede idealizar como una rama ascendente, asociada a una rigidez elástica inicial, hasta que ocurre el primer agrietamiento inclinado, y continua con una menor pendiente, debido a la propagación del agrietamiento inclinado en la superficie de la mampostería, hasta que alcanza su capacidad máxima ante cargas laterales (resistencia). A partir de este punto se puede observar un deterioro en la resistencia asociado al daño progresivo en los extremos de los castillos, entre otros factores. Asimismo, se ha observado que la apertura y el cierre de grietas diagonales provocan un estrechamiento de los lazos histeréticos cerca del origen, limitando la capacidad de disipación de energía. Por otra parte, en especimenes con refuerzo horizontal interior y con refuerzo exterior se ha observado un incremento en la resistencia ante cargas laterales y en la capacidad de deformación respecto a los especimenes de mampostería confinada. Este incremento depende, principalmente, de la cuantía y el detallado del refuerzo empleado. Finalmente, la participación del refuerzo disminuyó la tasa de deterioro de resistencia, en comparación con la observada en especimenes de mampostería confinada.

\section{Modelos analíticos}

Con base en un extenso programa de investigación experimental, Meli (1975) propuso uno de los primeros modelos analíticos para representar las características carga-deformación de estructuras de mampostería confinada. El modelo propuesto consiste de una envolvente trilineal y 9 parámetros que definen el comportamiento de muros de mampostería confinada y confinada con refuerzo interior. Moroni y colaboradores (1994) propusieron un modelo analítico para representar el comportamiento histerético de estructuras de mampostería confinada, el cual se caracteriza por tener una envolvente trilineal y ciclos histeréticos con degradación de rigidez. Sin embargo, debe notarse que el modelo propuesto no puede reproducir el deterioro de resistencia una vez alcanzada la carga máxima y, por consiguiente, la energía histerética disipada en cada ciclo, lo cual le resta fidelidad para representar el comportamiento observado en ensayes de laboratorio. Como parte de un amplio programa de investigación sobre la vivienda económica en México, Flores y Alcocer (1996) propusieron un modelo analítico para reproducir el comportamiento de estructuras de mampostería confinada. El modelo esta caracterizado por una envolvente trilineal y permite reproducir la degradación de rigidez en función de la rigidez inicial y el nivel de desplazamiento máximo alcanzado en los ciclos previos a la descarga, así como el deterioro de resistencia. Recientemente, Tomazevic y Lutman (1996b) emplearon el modelo analítico propuesto por Park y colaboradores (1987), conocido en la literatura como el modelo de tres parámetros, para reproducir la respuesta histerética de 32 especimenes de mampostería con refuerzo interior. Este modelo analítico (ver figura 1) fue propuesto originalmente para reproducir el comportamiento histerético de estructuras de concreto reforzado mediante la selección adecuada de tres parámetros. Cada parámetro controla el nivel de deterioro de resistencia (parámetro $\mathrm{HB}$ ), de degradación de rigidez (parámetro $\mathrm{HC}$ ), y del adelgazamiento de los lazos histeréticos cerca del origen (parámetro HS), respectivamente. Los autores propusieron una metodología para determinar el valor de cada parámetro y valores promedio que permitieran reproducir el comportamiento observado de los especimenes de mampostería ensayados previamente. Sin embargo, debe notarse que el modelo de tres parámetros no permite reproducir 
de forma gradual el deterioro de resistencia, dando como resultado lazos histeréticos poco representativos de especimenes de mampostería sometidas a cargas laterales cíclicas reversibles. Por ello, en este estudio se utilizó una versión modificada por Cheok y colaboradores (1998) del modelo de tres parámetros. La versión modificada del modelo analítico reproduce la degradación de resistencia ante cargas laterales mediante la selección apropiada de dos parámetros que dependen del nivel de deformación inelástica (parámetro HBD) y de la energía disipada acumulada en cada ciclo (parámetro HBE). Una descripción detallada del modelo analítico empleado en este estudio puede encontrarse en (Park et al., 1987; Cheok et al., 1998).

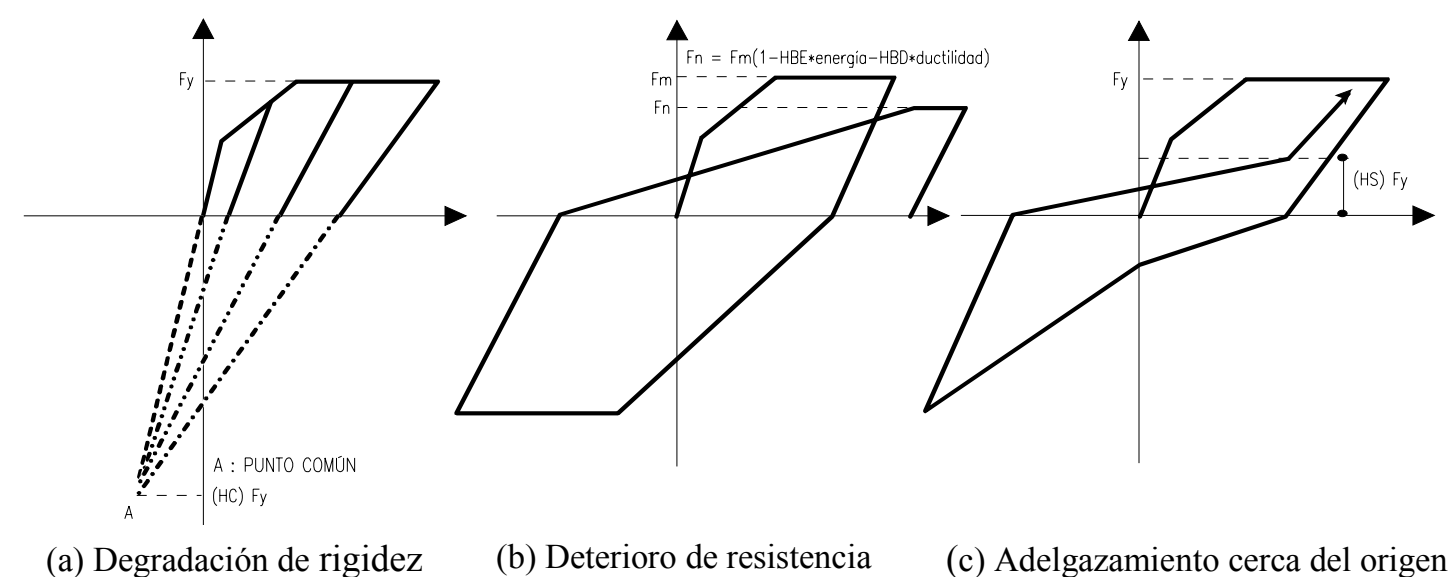

Figura 1. Efecto de los tres parámetros en la respuesta analítica (Park et al., 1987).

\section{Calibración del modelo analítico}

Con la intención de reproducir analíticamente el comportamiento observado de muros y estructuras de mampostería durante ensayes de laboratorio, se recopiló la respuesta cargadeformación de 14 especimenes de mampostería (4 construidos de mampostería confinada, 4 construidos de mampostería confinada con refuerzo interior, 2 de mampostería con refuerzo interior y 4 construidos de mampostería confinada con refuerzo exterior) que habían sido ensayados previamente en programas de investigación desarrollados en México y Eslovenia. Una descripción detallada de la geometría, detalles del refuerzo, calidad de los materiales, respuesta histerética y modo de falla de los especimenes ensayados pueden encontrarse en la literatura (Alcocer y Meli, 1995; Alcocer et al., 1996; Aguilar et al., 1996; Tomazevic et al., 1996a). Los 12 especimenes de mampostería que se ensayaron en México se construyeron con piezas macizas tipo artesanal de barro rojo recocido y fueron confinados mediante dalas y castillos de concreto reforzado. En los muros con refuerzo interior se emplearon alambres corrugados de alta resistencia estirados en frío embebidos a lo largo de la superficie de mortero, mientras que en los muros con refuerzo exterior se empleo malla de alambre electrosoldada anclada en la superficie de los paneles. Los 2 especimenes ensayados en Eslovenia se construyeron con dos tipos de bloques huecos de concreto y fueron reforzados interiormente con alambres corrugados de alta resistencia entre las hiladas de las piezas. 
De esta manera, el modelo analítico y los parámetros que controlan la respuesta histerética fueron cuidadosamente calibrados, mediante un proceso iterativo para minimizar la diferencia entre la respuesta medida y la calculada. En general, la calibración de cada espécimen fue satisfactoria. En la figura 2 se muestra la respuesta experimental y analítica del espécimen M-1/4E6 ensayado por Aguilar y colaboradores (1996). Este espécimen fue reforzado horizontalmente con dos alambres corrugados de $6.3 \mathrm{~mm}$ de alta resistencia estirados en frío colocados horizontalmente entre las juntas de mortero. El espécimen exhibió una gran capacidad de deformación y de disipación de energía una vez alcanzada la carga máxima. El modo de falla se caracterizó por el agrietamiento horizontal a lo largo de las hiladas que fueron reforzadas. Para mostrar la versatilidad del modelo analítico, en la figura 3 se presenta una comparación semejante para el espécimen 3DR (Ruiz, 1995). El espécimen 3DR fue un modelo tridimensional de dos niveles, a escala natural, de mampostería confinada rehabilitado mediante el uso de malla de alambre y recubrimiento de mortero. Una vez que fueron cuidadosamente calibrados los parámetros que permitían reproducir la respuesta histerética de cada espécimen se eligieron parámetros representativos para cada tipo de mampostería: mampostería confinada, mampostería con refuerzo interior y mampostería con refuerzo exterior.
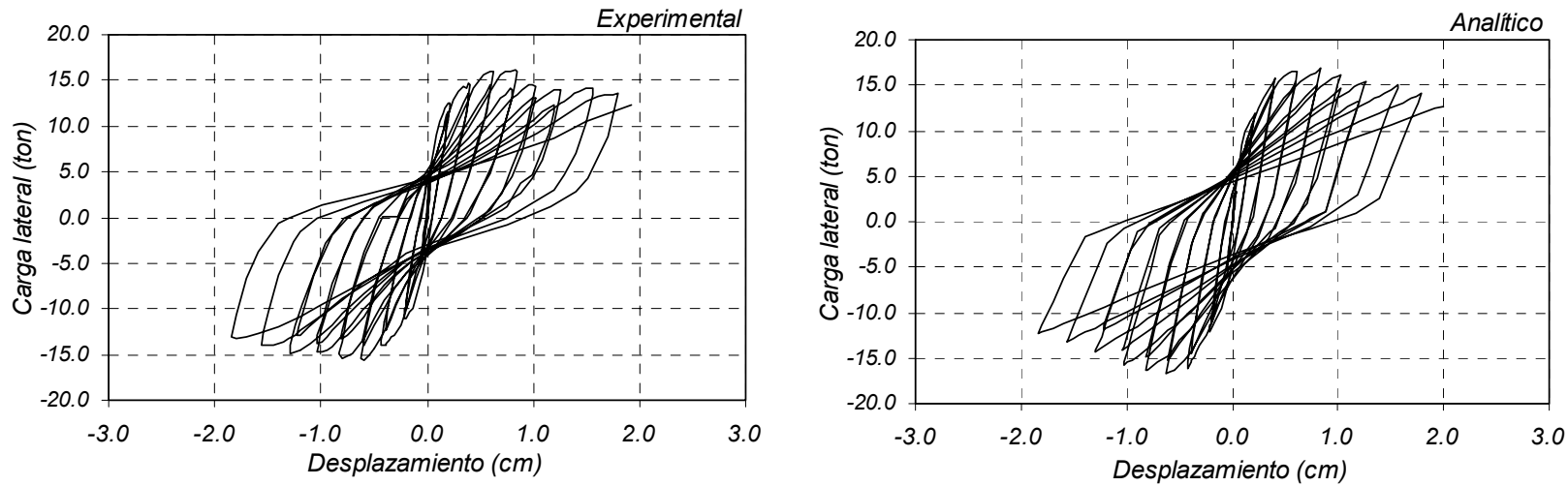

Figura 2. Comparación entre la respuesta experimental y analítica del modelo M-1/4-E6.
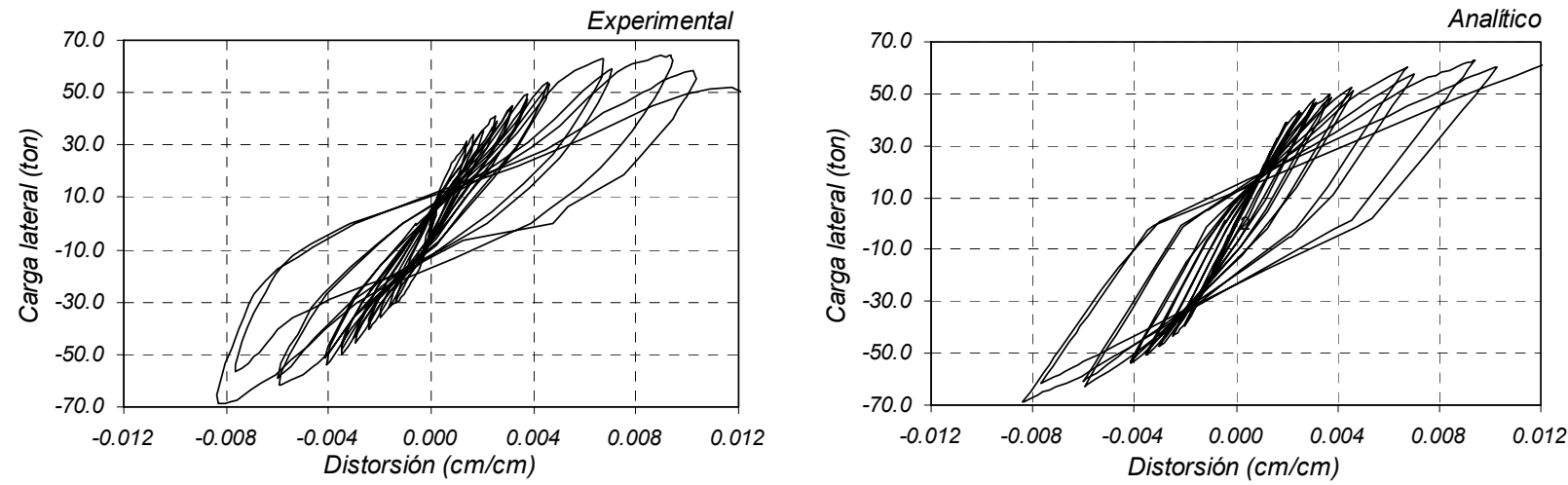

Figura 3. Comparación entre la respuesta experimental y analítica del modelo 3DR.

En la Tabla 1 se presentan los parámetros elegidos en este estudio para obtener la respuesta analítica de estructuras de mampostería. Debe mencionarse que los valores indicados en 
la Tabla 1 solo son representativos de los especimenes empleados para calibrar el modelo analítico y no intentan representar, en general, el posible comportamiento de estructuras de mampostería construidas con piezas provenientes de diferentes partes del país.

Tabla 1. Parámetros seleccionados para reproducir el comportamiento histerético

\begin{tabular}{lcccc}
\hline \multirow{2}{*}{ Tipo de mampostería } & \multicolumn{3}{c}{ Parámetros del modelo analítico } \\
\cline { 2 - 5 } & HC & HBD & HBE & HS \\
\hline Mampostería confinada & 3.5 & 0.060 & 0.060 & 0.015 \\
Mampostería con refuerzo interior & 3.0 & 0.025 & 0.025 & 0.015 \\
Mampostería con refuerzo exterior & 2.4 & 0.040 & 0.040 & 0.030 \\
\hline
\end{tabular}

\section{REGISTROS Y EVENTOS SÍSMICOS CONSIDERADOS}

En este estudio se utilizaron registros con las siguientes características: (1) registrados en estaciones acelerográficas sobre roca o terreno firme; (2) registrados en estaciones acelerográficas en las que existe información detallada de las condiciones geológicas y geotécnicas; (3) registrados en estaciones de terreno libre o bien en planta baja de edificios de poca altura y en la que los efectos de interacción suelo estructura fueran despreciables; (4) registrados en sismos con magnitudes de onda superficial $\left(\mathrm{M}_{\mathrm{s}}\right)$ superior a 5.7 ; y $(5)$ registros en las que al menos una de las componentes horizontales del terreno tiene una aceleración máxima superior a $40 \mathrm{~cm} / \mathrm{s}^{2}$.

Tabla 2. Resumen de los acelerogramas utilizados en este estudio.

\begin{tabular}{|c|c|c|c|c|c|c|}
\hline \multirow[b]{2}{*}{ Fecha } & \multirow[b]{2}{*}{ Sismo } & \multirow[b]{2}{*}{$\mathbf{M}_{\mathrm{s}}$} & \multicolumn{4}{|c|}{ Tipo de Terreno } \\
\hline & & & $\overline{A, B}$ & $\mathrm{C}$ & D & Total \\
\hline $21 / 07 / 57$ & Kern County & 7.7 & 0 & 4 & 2 & 6 \\
\hline $09 / 04 / 68$ & Borrego Mountain & 6.7 & 0 & 0 & 2 & 2 \\
\hline $09 / 02 / 71$ & San Fernando & 6.5 & 8 & 10 & 6 & 24 \\
\hline $15 / 10 / 79$ & Imperial Valley & 6.8 & 2 & 2 & 36 & 40 \\
\hline $24 / 04 / 84$ & Morgan Hill & 6.1 & 2 & 4 & 6 & 12 \\
\hline $08 / 07 / 86$ & Palm Springs & 6.0 & 8 & 0 & 0 & 8 \\
\hline $01 / 10 / 87$ & Whittier & 6.1 & 4 & 18 & 22 & 44 \\
\hline $04 / 10 / 87$ & Whittier Aftershock & 6.1 & 2 & 0 & 0 & 2 \\
\hline $17 / 10 / 89$ & Loma Prieta & 7.1 & 26 & 22 & 16 & 64 \\
\hline $28 / 06 / 91$ & Sierra Madre & 5.8 & 4 & 0 & 0 & 4 \\
\hline $28 / 06 / 92$ & Landers & 7.5 & 4 & 2 & 16 & 22 \\
\hline \multirow[t]{2}{*}{$17 / 01 / 94$} & Northridge & 6.8 & 18 & 14 & 4 & 36 \\
\hline & Total & & 78 & 76 & 110 & 264 \\
\hline
\end{tabular}

Con base en este criterio de selección se escogieron 264 acelerogramas registrados en el estado de California (Estados Unidos) durante 12 sismos históricos. Los movimientos sísmicos fueron divididos en tres grupos de acuerdo a las condiciones geológicas de cada estación seleccionada. El primer grupo consistió de acelerogramas registrados en estaciones ubicadas 
sobre roca, con velocidades de onda de corte promedio mayores que $760 \mathrm{~m} / \mathrm{s}$. El segundo grupo consistió de historias de aceleración obtenidas en estaciones localizadas sobre rocas suaves o suelos firmes con velocidades de onda de corte promedio entre 360 y $760 \mathrm{~m} / \mathrm{s}$. Las estaciones acelerográficas del primer grupo corresponden a tipos de terreno clasificados como A y B de acuerdo a las recomendaciones de diseño americanas (FEMA 273, 1997), mientras que las estaciones acelerográficas del segundo y tercer grupo corresponden a tipos de terreno $\mathrm{C}$ y $\mathrm{D}$, respectivamente. En la Tabla 2 se resume el número de acelerogramas de cada sismo correspondiente a cada tipo de terreno y en la figura 4 puede verse la distribución de magnitud e intensidad (por ejemplo, aceleración máxima del terreno) con respecto a la distancia de los acelerogramas seleccionados. En la figura 5 puede observarse la forma espectral elástica de aceleración promedio calculada $(\zeta=5 \%)$ para cada tipo de terreno.
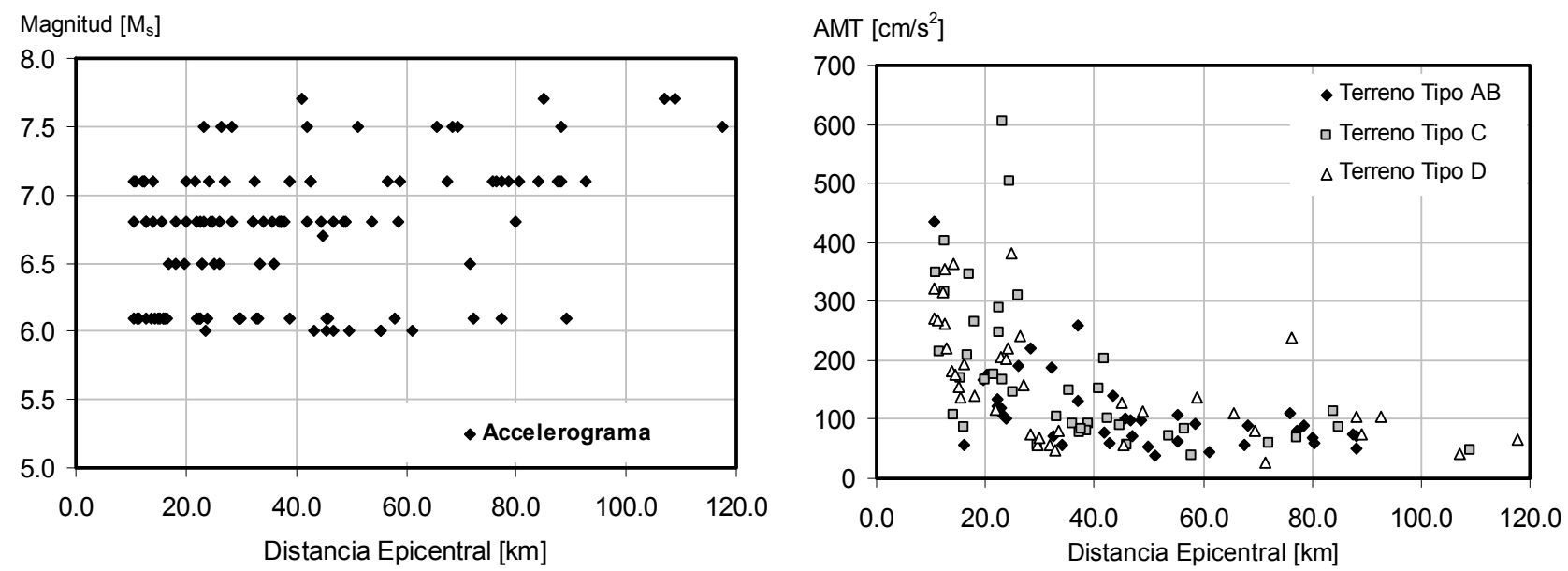

Figura 4: a) Distribución de la magnitud y distancia epicentral considerada en este estudio; b) Distribución de intensidad y distancia epicentral.

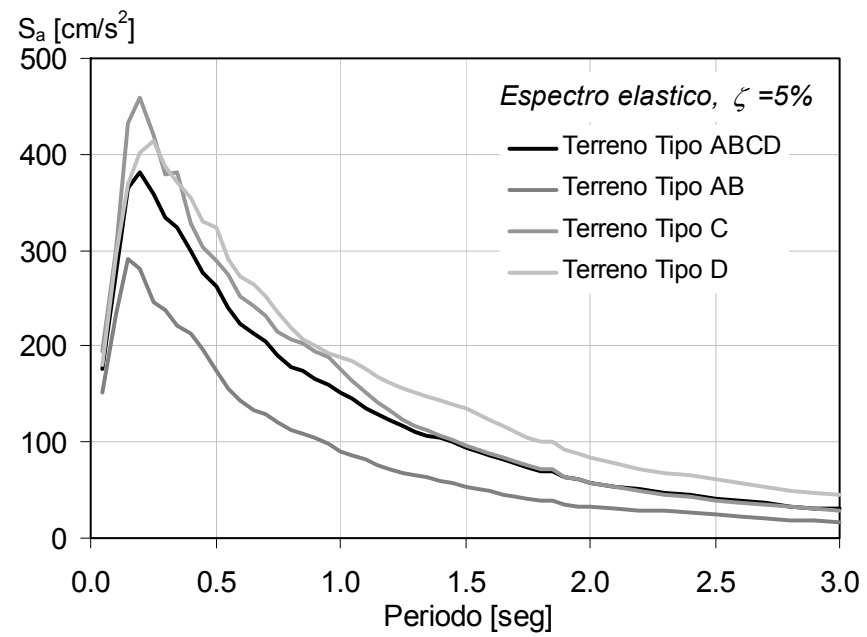

Figura 5. Espectro de respuesta elástico promedio. 


\section{PROCEDIMIENTO DE ANÁLISIS}

Como se mencionó anteriormente, la mayoría de los estudios previos sobre los factores de reducción de resistencia por ductilidad han considerado un comportamiento elastoplástico perfecto (Miranda y Bertero, 1994). Sin embargo, es evidente que este modelo no considera el fenómeno de la degradación de rigidez, el deterioro de resistencia y la disminución en la capacidad de disipación de energía histerética. Con la intención de estudiar la influencia de la degradación estructural en las demandas de resistencia lateral, en este estudio se empleó el modelo bilineal para representar a las estructuras que no presentan degradación estructural y el modelo histerético calibrado para cada tipo de mampostería para representar a las estructuras que exhiben degradación estructural. En la figura 6 se muestra la respuesta inelástica de un sistema de un grado de libertad, con periodo de vibración de $0.2 \mathrm{seg}$. y una misma demanda de ductilidad de cuatro, con ambos modelos al ser sometido a una historia de aceleraciones registrada en la estación 58505 (componente 190) durante el sismo de Loma Prieta. Es evidente la diferencia de comportamiento.
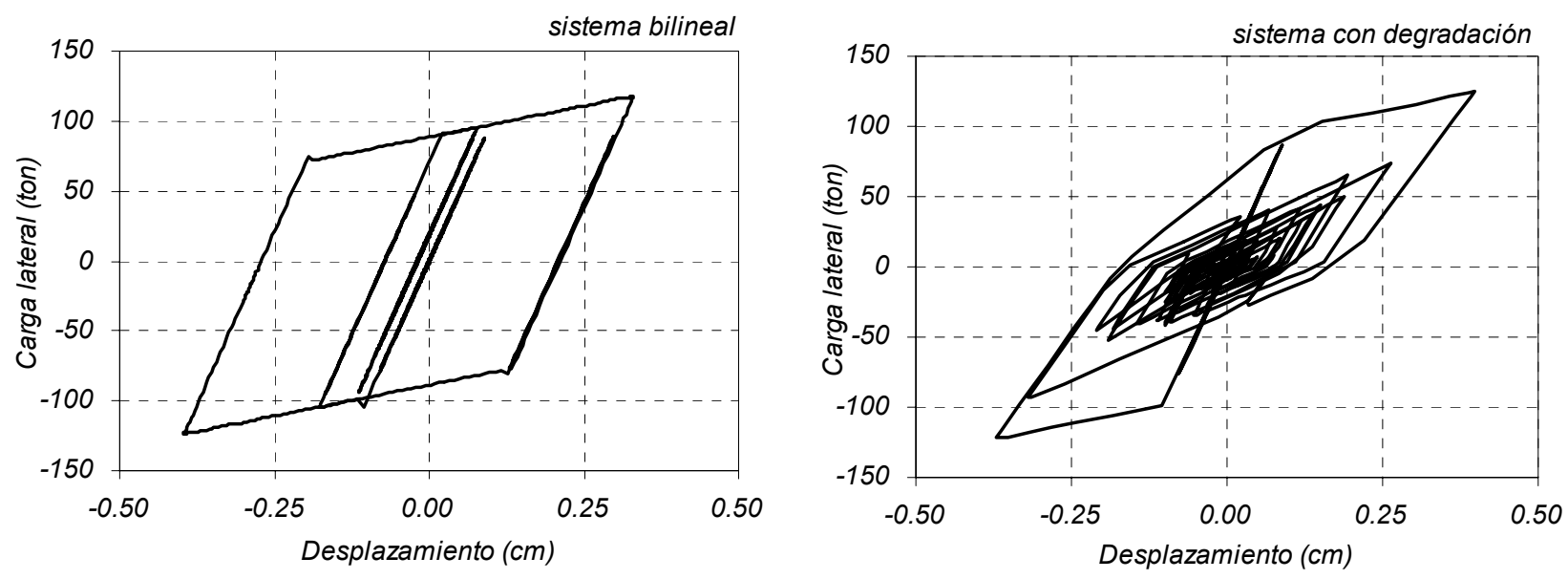

Figura 6. Comparación entre la respuesta del sistema bilineal y con degradación estructural.

El proceso analítico consistió en calcular las demandas de resistencia lateral inelástica para sistemas de un grado de libertad con cuatro tipos de comportamiento (tres con degradación estructural representativos de estructuras de mampostería y uno con comportamiento bilineal que es representativo de estructuras que no exhiben degradación estructural). En este estudio se considero una rigidez post-elástica igual al $9 \%$ de la rigidez inicial para el sistema bilineal y los sistemas con degradación estructural, la cual es representativa de los especimenes considerados en este estudio. En estructuras de mampostería, esta pendiente representa la rigidez posterior al primer agrietamiento inclinado. En la solución de la ecuación de movimiento dinámico se consideró un factor de amortiguamiento, $\zeta$, de $5 \%$. Para cada acelerograma se calculó la respuesta lateral de 15 sistemas de un grado de libertad con periodos de vibración, $T$, que cubrían un intervalo de periodos de $0.05 \mathrm{seg}$. a $1.0 \mathrm{seg}$. Cabe mencionar que el intervalo de periodos naturales de vibración en estructuras de mampostería es mas estrecho (menor a $0.5 \mathrm{seg}$.) según se ha evidenciado a partir de pruebas de vibración ambiental (Muria-Vila et al., 1989). Sin embargo, en este estudio se decidió utilizar un intervalo espectral más amplio y, de esta manera, poder 
identificar alguna tendencia significativa. Posteriormente, mediante un proceso iterativo, para cada acelerograma y para cada periodo natural de vibración del sistema se obtuvo la resistencia lateral requerida, $F_{y}\left(\mu=\mu_{i}\right)$, para alcanzar una demanda de ductilidad $\mu_{i}$ prefijada, considerando una tolerancia de $1 \%$ en la estimación. En este estudio se consideraron cinco valores de $\mu$ : 1 (respuesta elástica), 1.5, 2, 3 y 4 (El comportamiento observado de los muros y estructuras de mampostería considerados en este estudio mostró que las estructuras de mampostería bien detalladas y con adecuado confinamiento pueden alcanzar dichas capacidades de deformación inelástica). En cada iteración, la respuesta dinámica se obtuvo mediante un proceso de integración numérica empleando el método de aceleración lineal, considerando un incremento de tiempo variable en función del periodo de vibración del sistema con la finalidad de disminuir el error en la estimación en la respuesta cuando ocurre un cambio de rigidez en el sistema. Una vez determinadas las demandas de resistencia lateral elástica e inelástica, se calcularon los factores de reducción de resistencia utilizando la ecuación (1), para cada nivel de ductilidad determinado. Para fines de este estudio, se denomino $R_{\mu S D}$ a los factores de reducción de resistencia para sistemas sin degradación estructural y $R_{\mu C D}$ a los factores de reducción de resistencia por ductilidad para sistemas con degradación estructural.

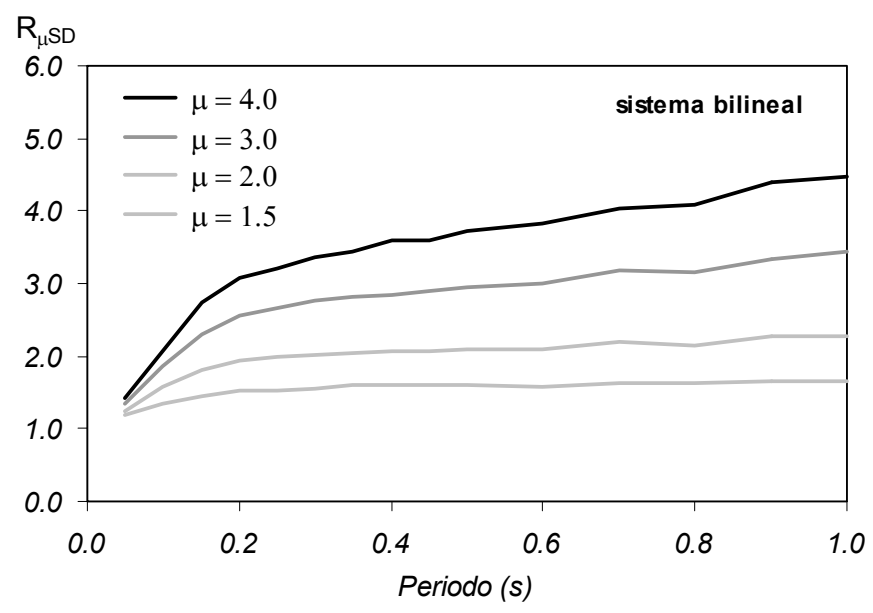

Figura 7. Media de los factores de reducción de resistencia por ductilidad (sistema sin degradación estructural).

\section{RESULTADOS DEL ESTUDIO ESTADÍSTICO}

\section{Tendencia central de $\mathbf{R}_{\mu}$}

En este estudio se calcularon 79,200 factores de reducción de resistencia $R_{\mu}$, correspondientes a 264 acelerogramas, 15 periodos, 5 niveles de demanda de ductilidad, y 4 tipos de comportamiento histerético. En la figura 7 se muestra la media muestral de los factores de reducción de resistencia calculados con el sistema sin degradación estructural, $R_{\mu S D}$, y en la figura 8 el factor de reducción de resistencia calculado para dos de las modalidades de refuerzo (mampostería confinada y con refuerzo interior), $R_{\mu C D}$. En general, los factores de reducción 
dependen del periodo de vibración del sistema, $T$, del nivel de deformación inelástico, $\mu$, y del tipo de comportamiento histerético. Puede observarse que para sistemas con periodo corto $(0.05$ seg. $<T<0.5$ seg.) el factor de reducción $\left(R_{\mu C D}\right.$ o $\left.R_{\mu S D}\right)$ es menor que el factor de ductilidad, $\mu$, mientras que para periodos intermedios $(0.5 \mathrm{seg}$. $<T<1.0 \mathrm{seg}$.) los factores de reducción tienden a ser ligeramente superiores a $\mu$. En particular, debe notarse que para sistemas de periodo corto ( $T$ $<0.5$ seg.) los factores de reducción $R_{\mu}$ se incrementan no linealmente conforme aumenta el nivel de deformación inelástica $\mu$ hasta un periodo de vibración límite a partir del cual el factor de reducción $R_{\mu}$ es ligeramente superior al factor de ductilidad $\mu$. Este periodo limítrofe depende del factor de ductilidad $\mu$.
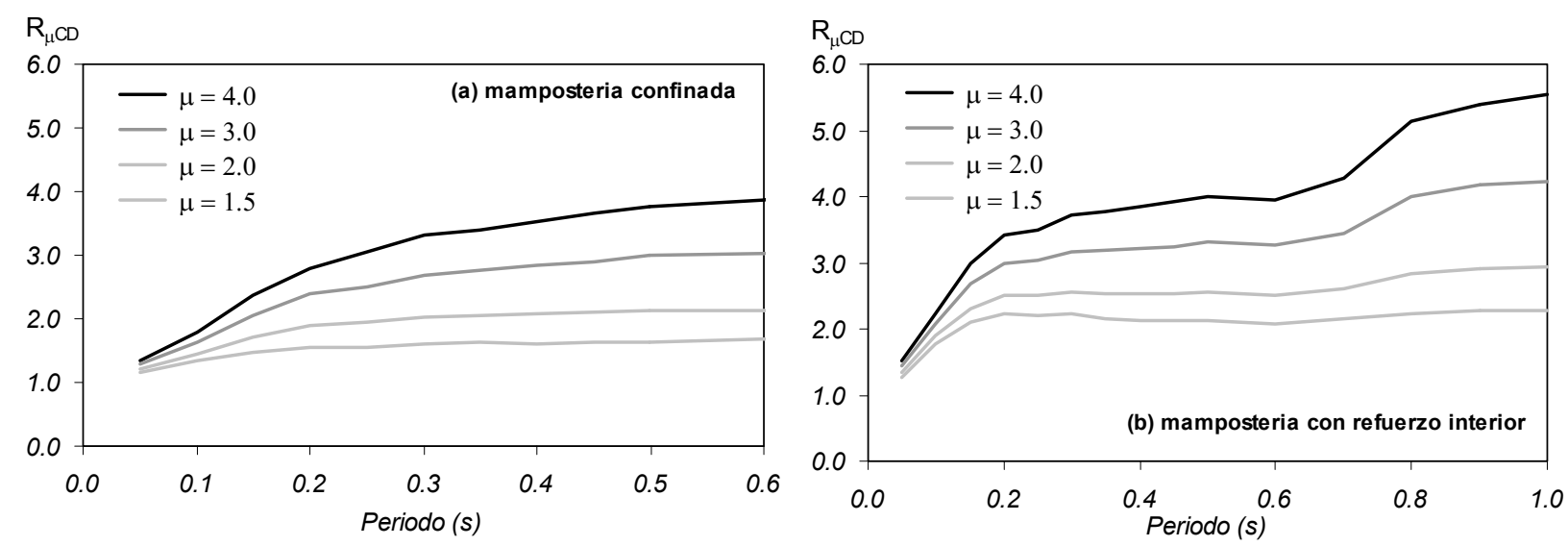

Figura 8. Media de los factores de reducción de resistencia por ductilidad (sistemas con degradación estructural).

\section{Modelo probabilista de $\mathbf{R}_{\mu}$}

Una consideración de la incertidumbre en el diseño sismorresistente requiere un enfoque probabilista y, en consecuencia, se requiere conocer el modelo probabilista que mejor estima la demanda de resistencia lateral calculada. La dispersión en la resistencia lateral se atribuye, principalmente, a la incertidumbre inherente a la excitación sísmica (i.e., incertidumbre en la intensidad, contenido de frecuencia y duración del movimiento del terreno). Miranda (1993) observó que la función de densidad de probabilidad del tipo lognormal es adecuada para calcular la probabilidad de excedencia de los factores de reducción por ductilidad, $R_{\mu}$, calculada para sistemas con comportamiento elastoplástico. Por ello, en este estudio se verificó, mediante la prueba de ajuste Kolmogorov-Smirnov (K-S), que la distribución acumulada de probabilidad del tipo lognormal también puede ser usada para estimar la probabilidad de excedencia de $R_{\mu C D}$ ). Así, se empleo el método de momentos (Benjamin y Cornell, 1970) en la estimación de parámetros probabilistas (por ejemplo, usando la media y la desviación estándar del logaritmo de los datos). En la figura 9(a) puede observarse la distribución de probabilidad empírica de $R_{\mu C D}$ y en la figura 9(b) se presenta el ajuste de los datos asumiendo una distribución de probabilidad del tipo lognormal para el caso de sistemas representativos de mampostería confinada. Finalmente, esta hipótesis se verificó para todo el rango de periodos estudiado. 

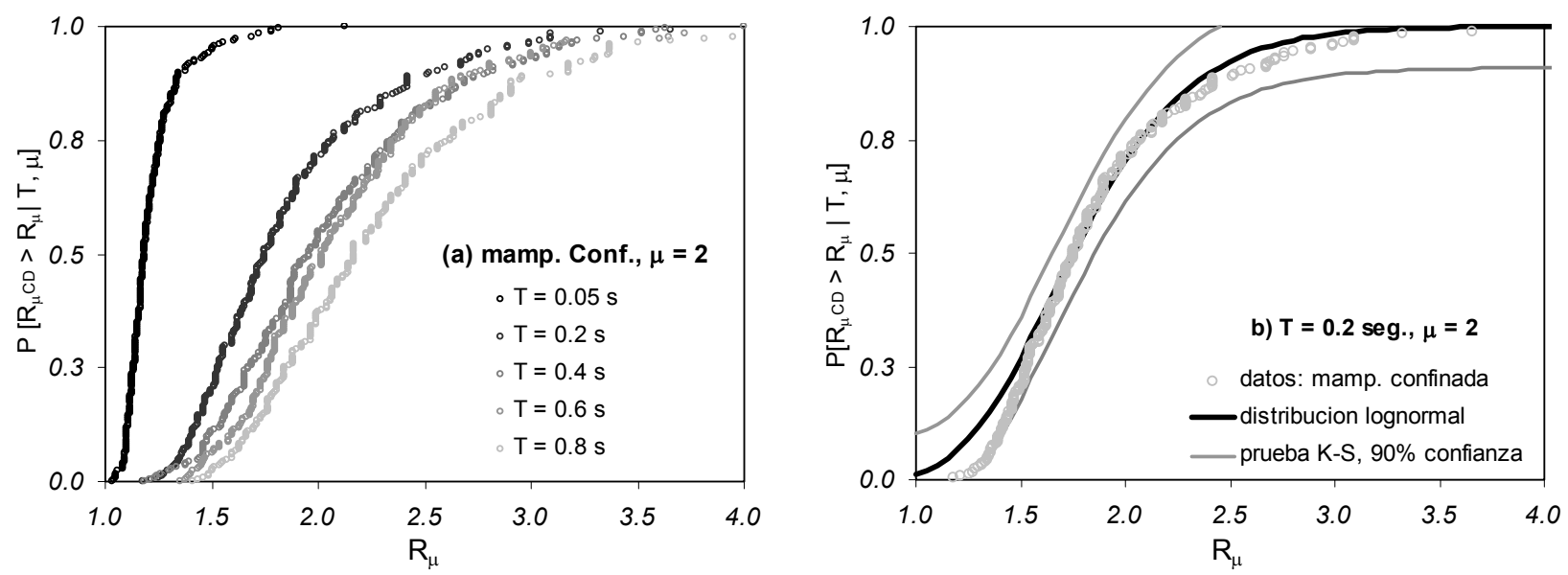

Figura 9. Distribución de probabilidades del factor de reducción de resistencia.

\section{Dispersión de $\mathbf{R}_{\mu}$}

Si bien la media de los factores de reducción de resistencia por ductilidad es muy importante, ya que representa una medida directa del valor esperado de estos factores, también es muy importante conocer la dispersión alrededor de la tendencia central (por ejemplo, media o mediana). En particular, es necesario cuantificar el nivel de dispersión y conocer los parámetros que influyen en incrementar o disminuir dicha dispersión. A continuación, se presentan algunas observaciones asociadas a los sistemas representativos de mampostería confinada.
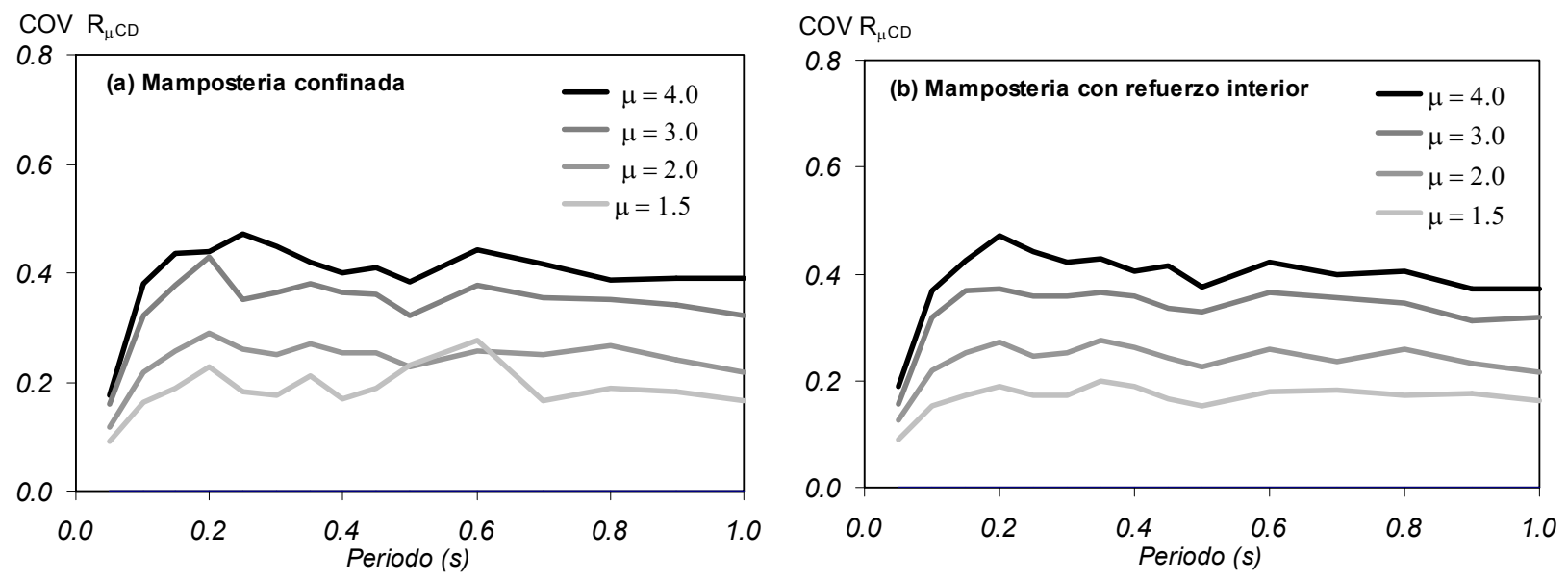

Figura 10. Dispersión del factor de reducción de resistencia por ductilidad.

Uno de los parámetros más comúnmente utilizados y efectivos para cuantificar la dispersión es el coeficiente de variación (COV), el cual se define como el cociente de la desviación estándar entre la media. Los coeficientes de variación de $R_{\mu C D}$ en función del periodo de vibración y del nivel de ductilidad, $\mu$, para dos tipos de comportamiento histerético se muestran en las figuras 10(a) y 10(b). Puede verse que, para un periodo dado, la dispersión se 
incrementa en la medida en la que el nivel de deformación inelástica, $\mu$, aumenta. Además puede verse que, con excepción de periodos muy cortos (menores a 0.2 seg.), para una demanda de ductilidad dada el coeficiente de variación no presenta variaciones importantes con cambios en el periodo de vibración. El coeficiente de variación para periodos de vibración entre 0.2 y $1.0 \mathrm{~s}$ varia aproximadamente de 0.20 para una ductilidad de 1.5 a 0.45 para una ductilidad de 4 , en promedio. En general, las observaciones anteriores también se aplican para los restantes tipos de comportamiento estudiados.

Otro de los parámetros usualmente empleados para cuantificar la dispersión consiste en calcular $R_{\mu C D}$ correspondiente a diferentes percentiles, los cuales proporcionan información de la probabilidad de exceder cierto valor de $R_{\mu C D}$ condicionado a un valor específico de ductilidad. En la figura 11 se presenta el valor de $R_{\mu C D}$, para estructuras de mampostería confinada, en función del periodo de vibración del sistema correspondiente a cinco percentiles $(\mathrm{p}=10,30,50,70$ y $90 \%)$ y dos niveles de ductilidad ( $\mu=2$ y 3 ). Por ejemplo, puede apreciarse que para una ductilidad de dos y un periodo de vibración de 0.2 seg., la mediana de $R_{\mu C D}$ es 1.75 (por ejemplo, existe $50 \%$ de probabilidad de exceder 1.75). Por otra parte, para el mismo periodo existe un $80 \%$ de probabilidad de que $R_{\mu C D}$ variara aproximadamente entre 1.41 y 2.53 (por ejemplo, $R_{\mu C D}$ se encuentra entre las curvas de los percentiles $10 \%$ y $90 \%$ ). De manera similar, en $40 \%$ de los casos (entre las curvas de $10 \%$ y $50 \%$ ) puede esperarse que el valor de $R_{\mu C D}$ sea mayor que $1.41 \mathrm{y}$ menor que 1.75. Al visualizar las figuras 10 (a) y (b) puede apreciarse que la dispersión es mayor para el sistema con ductilidad de 3 y que para este sistema la dispersión se incrementa al incrementarse el periodo de vibración. Esta observación es consistente con la tendencia observada para el coeficiente de variación de $R_{\mu C D}$.
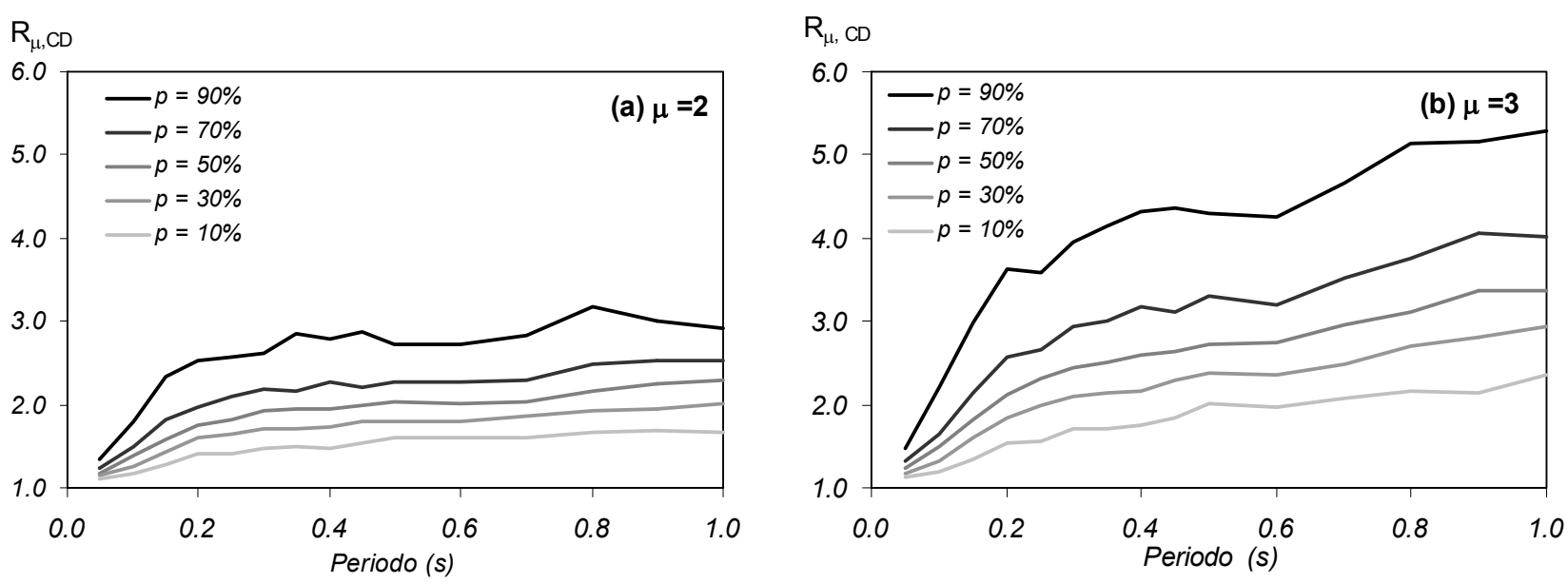

Figura 11. $R_{\mu C D}$ correspondientes a diferentes percentiles en función del periodo de vibración.

En la figura 12 se muestra el factor de reducción calculado para sistemas representativos de mampostería confinada $R_{\mu C D}$, en función del nivel de deformación inelástico, $\mu$, para un periodo de vibración de $0.2 \mathrm{seg}$. y correspondiente a cinco percentiles. Puede verse que para este periodo de vibración los factores de reducción se incrementan casi linealmente al incrementarse el nivel de deformación inelástica. Esta tendencia casi lineal es evidente no solamente para los valores de tendencia central $(\mathrm{p}=50 \%)$ si no también para valores grandes y pequeños de probabilidad de 
excedencia ( $\mathrm{p}=10 \%$ y 90\%). Asimismo, es importante notar una gran asimetría de la distribución de probabilidades conforme se incrementa el nivel de ductilidad. Es decir, conforme se incrementa el nivel de deformación inelástico de una estructura de mampostería confinada la dispersión respecto al valor central (mediana, $\mathrm{p}=50 \%$ ) del factor $R_{\mu C D}$ también se incrementa.

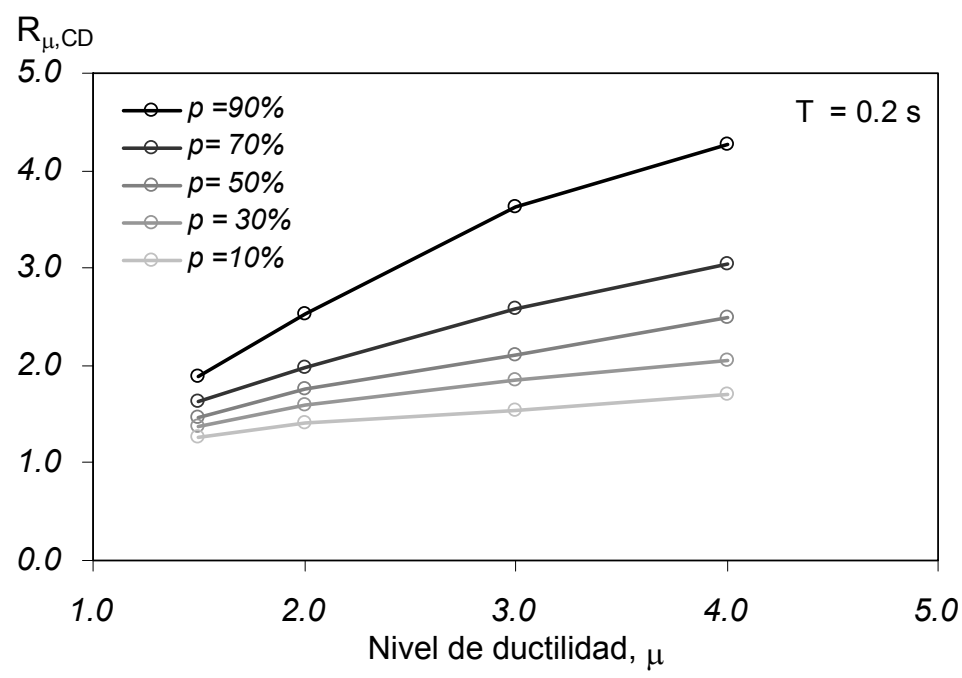

Figura 12. $R_{\mu C D}$ correspondientes a diferentes percentiles en función del nivel de ductilidad.

\section{Efecto de la degradación estructural en $\mathbf{R}_{\mu}$}

Con la finalidad de estudiar la influencia de la degradación estructural en las demandas de resistencia lateral se obtuvo, para cada registro, cada periodo y cada nivel de ductilidad, el cociente del factor de reducción obtenido con el modelo sin degradación estructural, $R_{\mu S D}$, y el factor de resistencia obtenido para cada tipo de mampostería (con degradación estructural), $R_{\mu C D}$.

$$
\frac{R_{\mu S D}}{R_{\mu C D}}=\frac{\frac{F_{y}(\mu=1)}{F_{y S D}\left(\mu=\mu_{i}\right)}}{\frac{F_{y}(\mu=1)}{F_{y C D}\left(\mu=\mu_{i}\right)}}=\frac{F_{y C D}\left(\mu=\mu_{i}\right)}{F_{y S D}\left(\mu=\mu_{i}\right)}
$$

Puede verse que este cociente es también igual al cociente de la resistencia lateral necesaria para controlar la demanda de ductilidad lateral en el sistema con degradación estructural a la del sistema bilineal (ec. 4).

Dicho cociente es muy útil, ya que permite emplear y modificar los resultados obtenidos en estudios previos de sistemas sin degradación estructural a fin de tener en cuenta la degradación estructural, como la que experimentan las estructuras de mampostería. Posteriormente se calculó el promedio de estos cocientes para cada periodo de vibración y para cada nivel de ductilidad 
estudiado. En la figura 13 se presenta la media del cociente $R_{\mu S D} / R_{\mu C D}$ para los 264 registros californianos y para cada modalidad de mampostería.
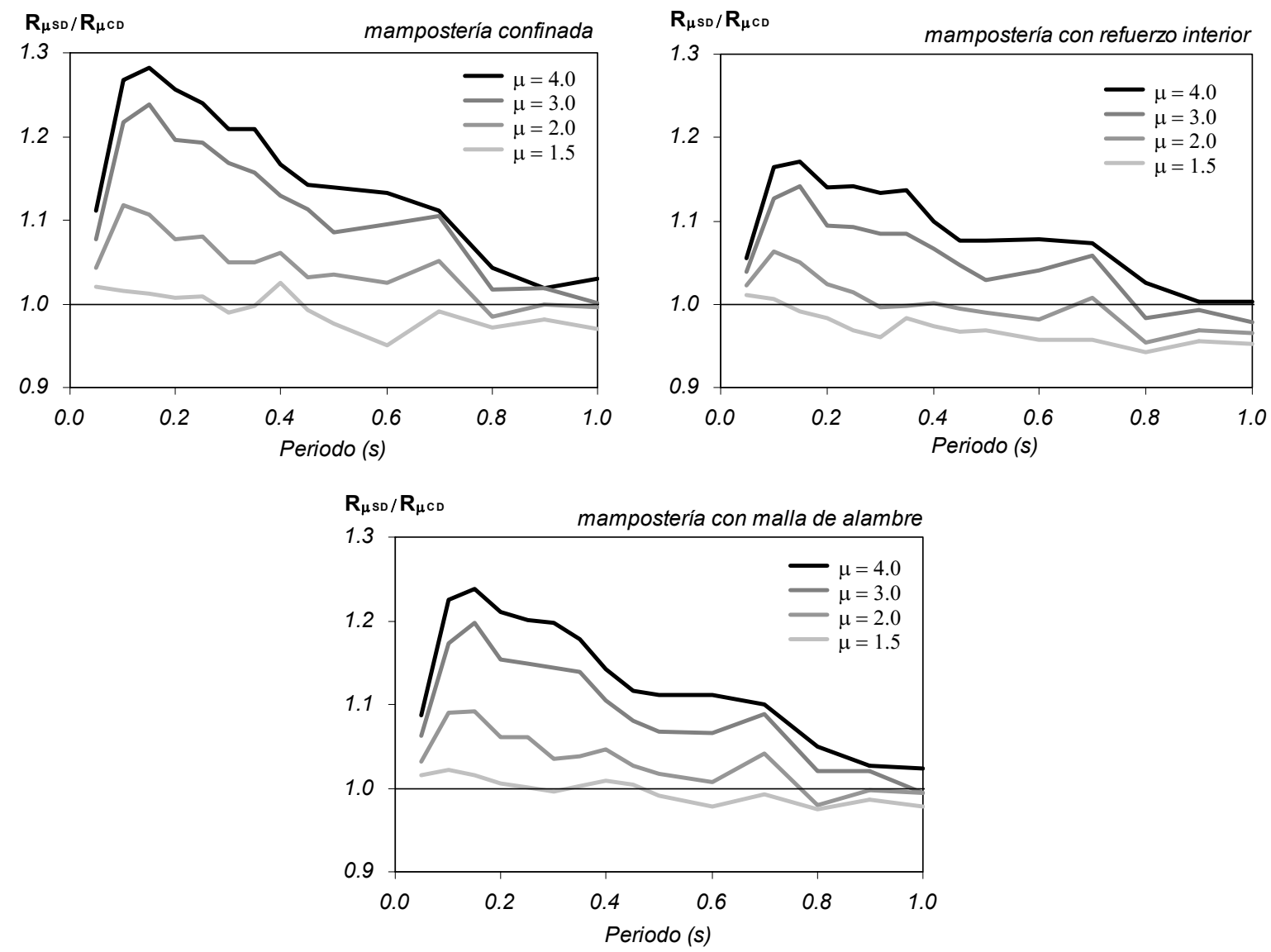

Figura 13. Promedio de los cocientes de los factores de reducción de resistencia sin considerar y considerando degradación estructural.

En las tres figuras puede observarse que para periodos de vibración pequeños a intermedios, hasta de $1.0 \mathrm{~s}$, este cociente es mayor a uno, o sea que el factor de reducción de resistencia de sistemas con degradación es menor al factor de resistencia de sistemas sin degradación. Esto implica que en esta región espectral para controlar la demanda de ductilidad a un mismo valor las estructuras que presentan degradación estructural, como las de mampostería, deberían diseñarse para fuerzas laterales mayores que aquellas que no presentan degradación estructural. En esta región espectral la degradación estructural no debería despreciarse. En la misma figura puede verse que dicho incremento necesario en resistencia lateral depende del nivel de ductilidad (por ejemplo, entre mayor sea la demanda de ductilidad que se permita en la estructura mayor deberá ser la resistencia de estructuras con degradación estructural respecto a las de estructuras sin degradación estructural), del periodo de vibración de sistema y de la modalidad de refuerzo de la mampostería. Cabe notar que el valor de los cocientes refleja el nivel de deterioro en las propiedades estructurales ya que los máximos valores se obtuvieron para los sistemas que representan el comportamiento de estructuras de mampostería confinada (las cuales tienen una tasa deterioro de resistencia mayor respecto a las estructuras de mampostería con refuerzo interior y con malla de alambre). Para este tipo de estructuras, puede verse que para 
ductilidades altas (por ejemplo, de 4 ) el cociente $R_{\mu S D} / R_{\mu C D}$ se incrementa hasta valores de 1.28 lo que implica incrementar las resistencia de sistemas con degradación en un $28 \%$ respecto de la de sistemas sin degradación si se quiere limitar el mismo nivel de ductilidad. Para sistemas que reproducen el comportamiento de estructuras de mampostería reforzadas interiormente y con refuerzo exterior, este incremento requerido en resistencia alcanza valores de $18 \%$ y $24 \%$, respectivamente, para limitar la misma ductilidad de desplazamiento que en sistemas que no exhiben degradación estructural. En general, para una ductilidad de $1.5 \mathrm{y}$ en la zona de periodo muy corto $(T<0.2$ seg.) no se aprecia un incremento importante. Asimismo, puede observarse que para periodos de vibración mayores a $0.2 \mathrm{seg}$. el cociente $R_{\mu S D} / R_{\mu C D}$ disminuye conforme aumenta el periodo de vibración del sistema y que la zona que limita los cocientes mayores a uno de la de cocientes menores a uno no es constante sino que depende del nivel de ductilidad. Para los tres tipos de comportamiento, puede decirse que el periodo limítrofe disminuye conforme se incrementa la demanda de ductilidad.

\section{COMPARACIÓN CON PROPUESTAS NORMATIVAS}

En la actualidad, los requerimientos de análisis y diseño sísmico para estructuras de mampostería incluidos en la normatividad mexicana están basados en estudios experimentales y analíticos desarrollados en la década de los 70's (Meli, 1975) y actualizados a raíz de los eventos sísmicos de 1985 en la ciudad de México. Básicamente, las normas mexicanas para diseño sismorresistente de estructuras de mampostería siguen una filosofía de diseño por resistencia, considerando el uso de coeficientes sísmicos reducidos de diseño para evaluar las demandas de resistencia lateral. Dichos coeficientes reducidos consideran implícitamente la reducción por comportamiento inelástico. Recientemente, Ordaz y colaboradores (2003) propusieron modificaciones a la normatividad vigente en materia de diseño sísmico. Los autores recomiendan el uso de espectros elásticos suavizados de diseño cuyas ordenadas dependen principalmente del peligro sísmico asociado a sitios específicos de la ciudad de México, al periodo natural de vibración de la estructura, $T$, y al periodo predominante del movimiento del terreno, $T_{s}$. Para tener en cuenta el comportamiento inelástico de la estructura, las ordenadas elásticas son reducidas por el producto de dos factores que toman en cuenta la ductilidad, $Q$ ' (denominado $R_{\mu}$ en este estudio) y la sobrerresistencia inherente en cada estructura, $R$, respectivamente

$C(T, Q)=\frac{S a(T)}{R(T) Q^{\prime}(T, Q)}$

donde $C(T, Q)$ es la resistencia necesaria de diseño y $S_{a}(T)$ es la ordenada elástica de aceleración. El factor de reducción por ductilidad $Q$ ' depende explícitamente del periodo natural de vibración de la estructura y del nivel de deformación inelástica, $Q$ (denominado $\mu$ en este estudio) e implícitamente del cociente entre el desplazamiento espectral y el desplazamiento máximo del suelo. $Q$ ' queda definido para tres regiones espectrales como 


$$
\begin{aligned}
& Q^{\prime}= \begin{cases}1+\frac{Q-1}{\sqrt{k}} \frac{T}{T_{a}} ; & \text { si } T \leq T_{a} \\
1+\frac{Q-1}{\sqrt{k}} ; & \text { si } T_{a}<T \leq T_{b} \\
1+(Q-1) \sqrt{\frac{p}{k}} ; & \text { si } T>T_{b}\end{cases} \\
& p=k+(1-k)\left(\frac{T_{b}}{T}\right)^{2}
\end{aligned}
$$

donde $Q$ es la demanda de ductilidad (denominada $\mu$ en este estudio), $T$ es el periodo natural de vibración de la estructura asociada al primer modo, $k$ es un factor que depende del desplazamiento máximo del suelo y del desplazamiento máximo espectral, $T_{a} \mathrm{y} T_{b}$ son periodos característicos que depende de $T_{s}$, y $p$ es un factor que depende de $k, T_{b}$ y $T$. El factor $Q$ ' propuesto por Ordaz y colaboradores se basó en un extenso estudio estadístico de la respuesta inelástica de sistemas de un grado de libertad con comportamiento elastoplástico perfecto (con rigidez posterior a la fluencia igual a cero) sometidos a mas de 400 acelerogramas registrados en diferentes condiciones de terreno (Ordaz y Pérez-Rocha, 1998).
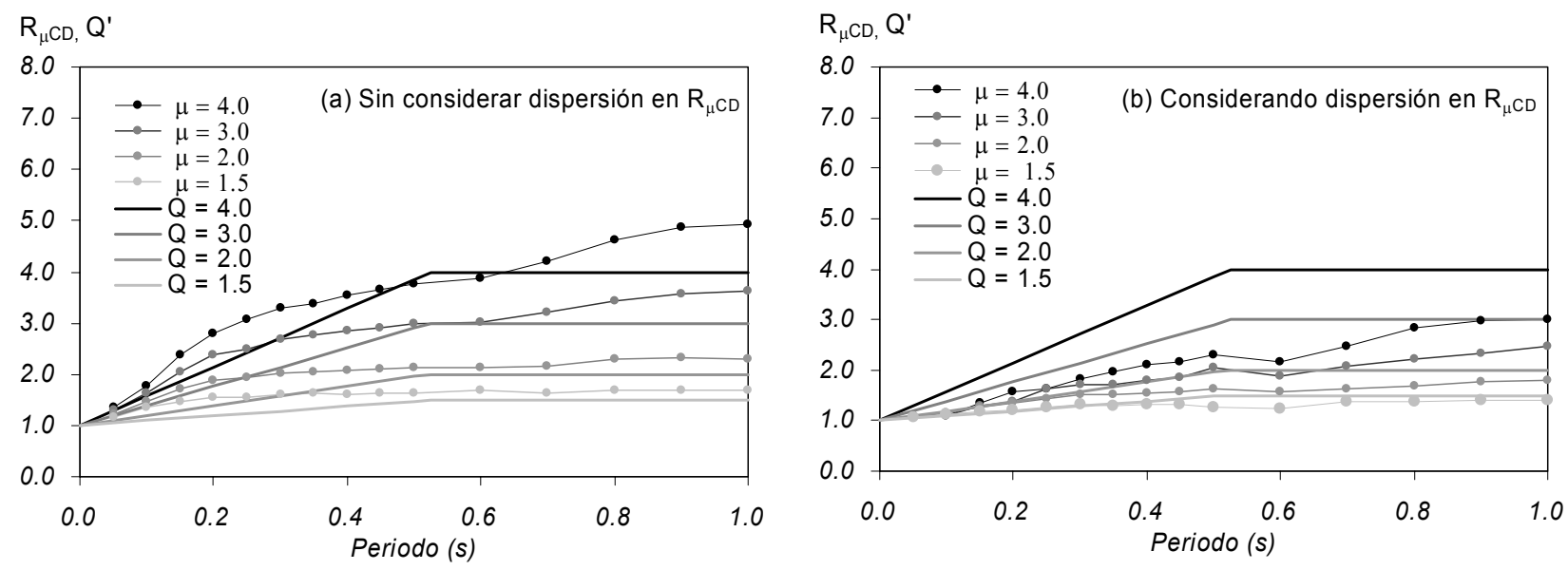

Figura 14. Comparación del factor de reducción de resistencia por ductilidad $R_{\mu C D} \mathrm{y}$ el factor de comportamiento sísmico Q'.

En la figura 14(a) se muestra una comparación de la media del factor de reducción de resistencia por ductilidad $R_{\mu C D}$, para estructuras de mampostería confinada, y el factor de reducción $Q^{\prime}$ (considerando $T_{s}=1 \mathrm{seg}$., $k=1 \mathrm{y} T_{a}=0.525 \mathrm{seg}$.) para cuatro niveles de deformación inelástica $(Q=\mu=1.5,2.0,3.0$ y 4.0). Como puede observarse, las ordenadas del factor $Q$ ' son menores que las ordenadas de $R_{\mu C D}$. Para el intervalo típico de periodos naturales de vibración en que se encuentran las estructuras de mampostería (0.05 seg. a $0.30 \mathrm{seg}$.), las ordenadas de $R_{\mu C D}$ son mayores (hasta en $35 \%$ para $T=0.2 \mathrm{seg}$.) que los valores propuestos para $Q$ ', sin importar el nivel de deformación inelástica. Esta observación sugiere que los valores 
propuestos para $Q$ ' conducen a fuerzas laterales de diseño mayores que si se emplean las ordenadas de $R_{\mu C D}$, por lo que su uso pudiera ser conservador y adecuado para una filosofía de diseño basada en resistencia. Por otra parte, es importante tener en tener en cuenta la dispersión en la estimación del factor de reducción de resistencia por ductilidad. Por ello, en la figura 14(b) se presenta la comparación del factor $R_{\mu C D}$ menos una desviación estándar (correspondiente a un percentil $=16 \%$ ) y el factor $Q$ '. Es evidente que si se toma en cuenta la dispersión en $R_{\mu C D}$, las ordenadas de $Q$ ' serian mayores que las ordenadas $R_{\mu C D}$ para todos los niveles de deformación inelástica, por lo que su uso conduciría a fuerzas laterales de diseño menores y, en consecuencia, a un escenario inadecuado.

Una posible explicación de las observaciones anteriores puede atribuirse a que los factores de reducción de resistencia por ductilidad, $R_{\mu}$, se incrementan conforme se incrementa el endurecimiento por deformación, o rigidez posterior a la fluencia, en la respuesta de sistemas de un grado de libertad. Nassar y Krawinkler (1991) observaron que las ordenadas de los factores de reducción por ductilidad calculados con modelos histeréticos que incluyen endurecimiento por deformación son mayores que las ordenadas de los factores de reducción de resistencia por ductilidad calculados con sistemas sin endurecimiento por deformación. Por ello, el uso de expresiones para calcular factores de reducción de resistencia por ductilidad derivadas de la respuesta de sistemas con comportamiento elastoplástico conduce a fuerzas laterales de diseño mayores que aquellas derivadas de sistemas con comportamiento histerético que incluyen endurecimiento por deformación (con o sin considerar el efecto de la degradación estructural). Cabe mencionar que el estudio de Moroni y colaboradores (1996) también observó que los factores de reducción de resistencia calculados para sistemas de múltiples grados de libertad, con un modelo que reproducía la degradación de rigidez, en estructuras de mampostería eran mayores que los valores calculados con la expresión propuesta en la normatividad chilena para determinar el factor de reducción de fuerzas sísmicas por ductilidad, $R^{*}$. (Riddell et al., 1989). Cabe notar que la expresión para calcular $R^{*}$ se basó en estudios analíticos de sistemas de un grado de libertad con comportamiento elastoplástico perfecto (i.e., sistemas que no exhiben degradación estructural).

Las observaciones anteriores indican que es de suma importancia fomentar el incremento de la rigidez post-elástica (i.e., posterior al primer agrietamiento) en el diseño de estructuras de mampostería en zonas sísmicas. Esto puede lograrse mediante el detallado del refuerzo, principalmente en los castillos confinantes, así como seguir un adecuado procedimiento constructivo. La importancia de los castillos radica en el confinamiento que proporcionan a los muros de mampostería ya que funcionan como un zuncho, evitando que los muros al agrietarse queden totalmente sueltos. Adicionalmente, los castillos contribuyen a mantener la capacidad ante cargas laterales y, mas aún, a incrementarla después del agrietamiento inicial. Asimismo, con una adecuada cantidad y distribución del refuerzo, los castillos permiten un aumento en la capacidad de deformación lateral de los muros y la disminución del deterioro de rigidez y de resistencia.

La autores quisieran notar que las observaciones vertidas en este inciso no implican que el efecto de la degradación estructural no sea importante para fines de diseño sismorresistente. Por ejemplo, la degradación estructural (i.e. deterioro de rigidez, degradación de resistencia y 
estrechamiento de los lazos de histéresis) es un factor muy importante cuando se evalúa el potencial de colapso de una estructura debido, principalmente, a inestabilidad dinámica, o bien en la estimación de las demandas de desplazamiento máximo inelástico. La evaluación del efecto de la degradación estructural adquirirá mayor relevancia cuando en la normatividad mexicana se quiera adoptar una filosofía de diseño basado en el desempeño estructural o en el control de los desplazamientos laterales en vez de la filosofía tradicional de diseño basada en resistencia lateral.

\section{CONCLUSIONES}

Se presentaron los resultados de un estudio analítico encaminado a estudiar los factores de reducción de resistencia $R_{\mu}$ aplicables a estructuras de mampostería. Este factor es de suma importancia para el diseño sismorresistente dado que permite obtener una estimación de la resistencia lateral necesaria para limitar la demanda de ductilidad en estructuras que se les permite incursionar en el intervalo inelástico durante eventos sísmicos intensos. Para tal fin, se empleo un modelo histerético muy versátil el cual fue cuidadosamente calibrado para reproducir el comportamiento observado en especimenes de mampostería ensayados en laboratorio. Así, se calcularon espectros inelásticos de demandas de resistencia lateral y espectros del factor de reducción de fuerzas laterales por comportamiento no lineal. Finalmente, se compararon los factores de reducción obtenidos con comportamiento bilineal con los obtenidos en este estudio y con el factor de reducción por ductilidad $Q$ ' propuesto recientemente para su uso en la normatividad mexicana. Las conclusiones derivadas de este estudio son las siguientes:

1. Mediante una selección apropiada de los parámetros que controlan la degradación de rigidez, el deterioro de resistencia y el adelgazamiento de los lazos de histéresis, es posible reproducir satisfactoriamente el comportamiento experimental de estructuras de mampostería empleando el modelo propuesto por Cheok y colaboradores (1998). Con el uso de este modelo, se pueden estimar las demandas de resistencia lateral de estructuras de mampostería con diferentes tipos de refuerzo.

2. Para estructuras de periodo corto $(T<0.5 \mathrm{seg}$.) el factor de reducción de resistencia por ductilidad $R_{\mu}$ es menor al factor de ductilidad, $\mu$, con una variación no lineal con el periodo de vibración. Asimismo, el factor de reducción se incrementa conforme se aumenta el nivel de ductilidad que puede tolerar la estructura. El periodo de vibración limítrofe a partir del cual el factor de reducción de resistencia es ligeramente superior al factor de ductilidad depende del nivel de deformación inelástica.

3. Los factores de reducción de resistencia para estructuras que exhiben degradación estructural, como las de mampostería, y periodos de vibración menores que $1.0 \mathrm{~s}$, son menores que los de estructuras sin degradación estructural. Esto tiene importantes implicaciones prácticas, sugiriendo que las estructuras de mampostería deberían ser diseñadas para fuerzas laterales mayores que las estructuras que no presentan degradación estructural, aun si tuvieran la misma capacidad de ductilidad. Es decir, en las estructuras que exhiben degradación estructural debería incrementarse su resistencia ante 
cargas laterales (i.e. del tipo sísmico) con respecto a la resistencia ante cargas laterales requerida para estructuras que no presentan degradación estructural.

4. El incremento adicional en resistencia lateral que requieren las estructuras de mampostería respecto a las estructuras que no exhiben degradación estructural depende de la ductilidad de desplazamiento, del periodo de vibración y del tipo de mampostería. Este incremento adicional es mayor para los sistemas que representan a las estructuras de mampostería confinada. En general, el incremento en resistencia lateral disminuye para periodos de vibración mayores que $0.2 \mathrm{~s}$ y es función del nivel de ductilidad así como del periodo de vibración del sistema.

5. La comparación de los resultados obtenidos en este estudio con los factores de reducción por ductilidad $Q$ ' recomendados para su uso en la normatividad mexicana (Ordaz et al., 2003) sugiere que el uso de $Q$ ' puede ser adecuado para una filosofía de diseño basada en la resistencia lateral. El uso de $Q$ ' conduce a fuerzas laterales de diseño mayores que si se comparan con la media de las ordenadas de $R_{\mu C D}$. Sin embargo, debe notarse que si se considera la dispersión en el valor medio de $R_{\mu C D}$, este factor conduciría a fuerzas de diseño mayores que las requeridas empleando $Q^{\prime}$.

\section{AGRADECIMIENTOS}

Los autores quisieran agradecer la revisión crítica y sugerencias constructivas vertidas por los dos revisores anónimos a beneficio del contenido de este artículo. Asimismo, el primer autor quisiera expresar su agradecimiento al Consejo Nacional de Ciencia y Tecnología por la beca-crédito otorgada para cursar sus estudios doctorales en la Universidad de Stanford bajo la supervisión del segundo autor. Finalmente, los autores reconocen la generosidad académica del Dr. Rodolfo Valles Mattox, quien proporcionó el código del modelo histerético empleado en este estudio, y del Dr. Roberto Meli Piralla, por las facilidades brindadas para la realización de esta investigación cuando fue director del CENAPRED.

\section{REFERENCIAS}

Alcocer, S M y R Meli (1995), "Test program on the seismic behavior of confined masonry walls", The Masonry Society Journal, Vol. 13, pp. 68-76.

Alcocer, S M, J Ruiz, J Pineda y J A Zepeda (1996), "Retrofitting of confined masonry walls with welded wire mesh", Memorias, $11^{\text {th }}$ World Conference on Earthquake Engineering, Artículo No. 1471, Acapulco, México.

Aguilar, G, R Meli, R Díaz y R Vázquez-del-Mercado (1996), "Influence of horizontal reinforcement on the behavior of confined masonry walls", Memorias, $11^{\text {th }}$ Eleventh World Conference on Earthquake Engineering, Artículo No. 1380, Acapulco, Mexico.

Bazán, E y R Meli (1980), "Seismic analysis of structures with masonry walls", Memorias, $7^{\text {th }}$ World Conference on Earthquake Engineering, Estambul, Turquía, Vol. 5, pp. 633-640. 
Benjamin, J R y C A Cornell (1970), "Probability, statistics, and decisions for civil engineers", McGraw-Hill Publishing Company, New York, NY.

Bertero, V V, J C Anderson, H Krawinkler y E Miranda (1991), "Design guidelines for ductility and drift limits: Review of state-of-the-practice and of-the-art on ductility and drift-based earthquake-resistant design of buildings", Report on Task1, A CUREe-Kajima Research Report.

Bertero, R D y V V Bertero (1999), "Redundancy in earthquake-resistant design”, ASCE Journal of Structural Engineering, Vol. 125, No. 4, pp. 438-444.

Cheok, G S, W C Stone y S K Kunnath (1998), "Seismic response of precast concrete frames with hybrid connections", ACI Structural Journal, Vol. 95, No. 5, pp. 527-539.

Federal Emergency Management Agency (1997), "NEHRP guidelines for the seismic rehabilitation of buildings," Reports FEMA 273 (Guidelines) and 274 (Commentary), Washington, D.C.

Flores, L E y S M Alcocer (1996), "Calculated response of confined masonry structures", Memorias, $11^{\text {th }}$ World Conference on Earthquake Engineering, Artículo No. 1830, Acapulco, Mexico.

Meli, R (1975), "Comportamiento sísmico de muros de mampostería”, Serie Azul No. 352, Instituto de Ingeniería, Universidad Nacional Autónoma de México, México, abril, 141 pp.

Miranda, E (1993), "Probabilistic site-dependent non-linear spectra", Earthquake Engineering \& Structural Dynamics, Vol. 22, No. 12, pp.1031-1046.

Miranda, E y V V Bertero (1994), "Evaluation of strength reduction factors for earthquakeresistant design”, Earthquake Spectra, Vol. 8, pp. 357-379.

Miranda, E (1997), "Strength reduction factors in performance-base design", Reporte $U C B / E E R C-97 / 05$, University of California, Berkeley.

Moroni, M O, M Astroza y S Tavonatti (1994), "Nonlinear models for shear failure in confined masonry walls", The Masonry Society Journal, Vol. 12, pp. 72-78.

Moroni, M O, M Astroza, J Gómez y R Guzmán (1996), "Establishing $\mathrm{R}_{\mathrm{w}}$ and $\mathrm{C}_{\mathrm{d}}$ factors for confined masonry buildings", ASCE Journal of Structural Engineering, Vol. 122, No. 10, pp. 1208-1215.

Muria-Vila, D, R Meli y R González-Alcorta (1989), “Dynamic behavior of masonry structures”, EERI 89-02, Lessons Learned from the 1985 Mexico Earthquake, Earthquake Engineering Research Institute, El Cerrito, California, pp. 188-191.

Nassar, A y H Krawinkler (1991), "Seismic demands of SDOF and MDOF systems", Report No. 95, John A. Blume Earthquake Engineering Center, Department of Civil and Environmental Engineering, Stanford University.

Ordaz, M, E Miranda, R Meli y J Avilés (2003), "New microzonation and seismic design criteria in the Mexico City building code", enviado para posible publicación en Earthquake Spectra.

Ordaz, M y L E Pérez-Rocha (1998), "Strength-reduction factors for elastoplastic systems: A new approach”, Earthquake Engineering and Structural Dynamics, Vol. 27, pp. 889-901. 
Tomazevic, M, M Lutman y L Petkovic (1996a), "Seismic behavior of masonry walls: Experimental simulation", ASCE Journal of Structural Engineering, Vol. 122, No. 10, pp. 10401047.

Tomazevic, M y M Lutman (1996b), "Seismic behavior of masonry walls: modeling of hysteretic rules”, ASCE Journal of Structural Engineering, Vol. 122, No. 10, pp. 1048-1054.

Tomazevic, M e I Klemenc (1997), "Seismic behavior of confined masonry walls", Earthquake Engineering and Structural Dynamics, Vol. 26, pp. 1059-1071.

Park, Y J, A M Reinhorn y S K Kunnath (1987), "IDARC: Inelastic damage analysis of reinforced concrete frame-shear wall structures", Technical Report NCEER-87-0008, National Center for Earthquake Engineering Research, State University of New York at Buffalo.

Riddell, R, P Hidalgo y E F Cruz (1989), "Response modification factors for earthquake resistant design of short period buildings", Earthquake Spectra, Vol. 5, No. 3, pp. 571-590.

Ruiz García, J (1995), "Reparación y refuerzo de una estructura tridimensional de mampostería confinada de dos niveles a escala natural", Tesis de Maestría, División de Estudios de Posgrado de la Facultad de Ingeniería, UNAM, 251 pp.

Uang, C-M (1991), "Establishing $\mathrm{R}$ ( or $\mathrm{R}_{\mathrm{w}}$ ) and $\mathrm{C}_{\mathrm{d}}$ factors for building seismic provisions", ASCE Journal of Structural Engineering, Vol. 117, No. 1, pp. 9-28.

Whittaker, A, G. Hart y C Rojahn (1999), "Seismic response modification factors", ASCE Journal of Structural Engineering, Vol. 125, No. 4, pp. 438-444. 